\title{
Real-Time MRI of Continent and Stress Incontinent Male Patients after Orthotopic lleal Neobladder
}

\author{
Ricarda M. Bauer ${ }^{a}$ Karin Herrmann ${ }^{b}$ Alexander Karl ${ }^{a} \quad$ Christian G. Stief ${ }^{a}$ \\ Yasmin Hocaoglu ${ }^{\text {a }}$ \\ Departments of ${ }^{\mathrm{a}}$ Urology and ${ }^{\mathrm{b}}$ Radiology, Ludwig Maximilian University, Munich, Germany
}

\section{Key Words}

Stress urinary incontinence $\cdot$ Neobladder $\cdot$ Real-time MRI •

Valsalva maneuver $\cdot$ Cystoprostatectomy

\begin{abstract}
Introduction: The aim of this study was to correlate anatomic differences with continence status in male patients after cystoprostatectomy and ileal neobladder using real-time magnetic resonance imaging. Patients and Methods: Anatomic differences of 14 male patients (7 daytime continent and 7 stress incontinent) with ileal neobladder were determined by measuring the orthogonal distance of the bladder neck to the pubococcygeal line (PCL) to correlate anatomic differences with continence status. Results: The median distance of the bladder neck to PCL was $+5.4 \mathrm{~mm}$ in continent patients before voiding whereas in incontinent patients it was $+2 \mathrm{~mm}(\mathrm{p}=0.012)$. During the Valsalva maneuver, the median distance in continent patients was +4 and in incontinent patients $-3 \mathrm{~mm}(\mathrm{p}=0.003)$. At the end of micturition, the median distance was $+2.3 \mathrm{~mm}$ in continent patients and $-12 \mathrm{~mm}$ in incontinent patients $(p=0.002)$. Conclusions: The bladder neck in incontinent patients showed more pronounced mobility in relation to the $P C L$ during micturition and the Valsalva maneuver as compared to continent patients. In addition, the ileal neobladder was positioned significantly lower in the pelvis of incontinent patients. These
\end{abstract}

preliminary results suggest that a stable bladder neck may be an important factor to reach full continence in patients with ileal neobladder.

Copyright $\odot 2011$ S. Karger AG, Basel

\section{Introduction}

In many centers worldwide, the orthotopic neobladder has become the standard care for bladder substitution after cystoprostatectomy in men. Despite modified surgical techniques - even in high-volume institutions - published postoperative stress urinary incontinence (SUI) rates are still up to $10 \%$ during daytime and up to $30 \%$ during nighttime [1]. Several factors including the construction of the reservoir, the compliance of the neobladder and the sphincter function play an important role in reaching full postoperative continence. Clinical experience shows however that even patients with orthotopic detubularized neobladder and good residual function of the sphincter often show SUI. For reduction of postoperative SUI and for further development of surgical techniques it is therefore necessary to improve and deepen

\section{R.M. Bauer and K. Herrmann contributed equally.}


our understanding of the physiological and anatomical processes in the neobladder and the lower pelvis.

Until today, imaging studies have focused mainly on staging, assessment of complications or follow-up issues and the description of different urinary diversions. Beyond this, magnetic resonance imaging (MRI) and functional dynamic (cine- or real-time) MRI in particular have been employed to assess functional pelvic floor disorders in the female pelvis and have gained importance as a valuable diagnostic adjunct in the workup of urinary dysfunctions $[2,3]$. Few preliminary data have been presented using real-time MRI to describe the postoperative configuration of neobladder [4].

The aim of this study was to evaluate the anatomic differences in continent and stress incontinent patients after cystoprostatectomy with orthotopic ileal neobladder using real-time MRI.

\section{Patients and Methods}

Fourteen men with orthotopic ileal neobladder with a median age of 69 years (mean 67.7, range 53-83) were enrolled in our study performed at a single institute. All patients had received nervesparing cystoprostatectomy with orthotopic ileal neobladder (58$59 \mathrm{~cm}$ terminal ileum, two surgeons with the same operative technique, $\mathrm{W}$-shaped pouch) for localized invasive urothelial cancer of the bladder. No patient exhibited metastatic disease or tumor recurrence. None of the patients underwent adjuvant or neoadjuvant chemoradiotherapy.

We investigated 7 daytime continent and 7 daytime stress incontinent males (2 with ICS grade 2, 4 with ICS grade 3, 1 with ICS grade 4) $[5,6]$. Patients were categorized in groups only based on daytime continence status since nighttime incontinence is caused by additional factors (e.g. timed voiding during the night, relaxed pelvic floor during sleeping). All daytime stress incontinent patients showed a positive cough test (bladder filling $\geq 150 \mathrm{ml}$ ) while the test was negative for all daytime continent patients. We analyzed the visible anatomic changes of the neobladder during the Valsalva maneuver via MRI, before and during voiding.

Before MRI, all patients had an intensive evaluation including urethroscopy for the assessment of the sphincter region and the anastomosis of bladder and urethra. In addition, we assessed daily pad use, pad weight in the standardized 1-hour pad test $[5,6]$, residual urine and performed a cough test and uroflowmetry. In urethroscopy, every patient showed a complete and concentric sphincter closure without sphincter defects during active sphincter contraction and no stenosis of the anastomosis region or the urethra. Time between surgery and MRI was at least 12 months (range 12-30, mean 18.4, median 17).

\section{Imaging}

Informed consent was obtained from all patients. One hour before MRI, all patients were requested to empty the bladder and to drink $200 \mathrm{ml}$ water in order to provide a consistently full neo- bladder during imaging. All patients underwent MRI on a 1.5-T MRI scanner (Magnetom Sonata; Siemens Medical Solutions, Erlangen, Germany) in the supine position using $\mathrm{T}_{2} \mathrm{~W}$ turbo spinecho or fast spinecho sequences in transversal and sagittal planes for anatomic detail and planning of the dynamic sequences. Functional dynamic real-time MRI (cine-MRI) was performed using steady-state free precession sequences (True FISP; Siemens Medical Solutions) in a single slice through the axis of the neobladder neck in sagittal orientation. Dynamic imaging was acquired at a frequency of 1 image/s before voiding, during the Valsalva maneuver and during micturition. In order to discriminate pelvic floor relaxation from other movements of the pelvic floor, patients were instructed to follow a certain protocol during the imaging procedure; patients were asked to perform first a voluntary contraction of the pelvic floor, typically inducing an upward movement of the levator ani muscle and afterwards to perform a Valsalva maneuver resulting in a downward movement of the levator ani muscle at MRI. Thus a 'neutral' position of the neobladder neck could be identified.

\section{Image Analysis}

In order to establish quantitative measures with regard to anatomical landmarks, the pubococcygeal line (PCL) was defined as a reference. The PCL is a virtual line extending from the inferior border of the pubic symphysis to the last joint of the coccyx. This line represents the level of the pelvic floor. Based on these, we measured the orthogonal distance of the neobladder neck to the PCL as established in studies on the female pelvis to evaluate the movement of the neobladder. Positive distances indicate a cranial position of the neobladder neck cranially to the PCL, whereas negative distances indicate a position below the PCL. In order to discriminate pelvic floor relaxation from other movements of the pelvic floor, patients were first instructed to perform pelvic floor contraction visualized as an upward movement of the levator ani muscle. Afterwards, patients were asked to perform a Valsalva maneuver visualized as a downward movement of the levator ani muscle.

\section{Statistics}

For statistical analysis, the software SPSS Statistics (SPSS 18.0; SPSS Inc., Chicago, Ill., USA) was used. The Mann-Whitney U test was used to test for significance. $p$ values $<0.05$ were regarded as significant.

\section{Results}

Based on patients' baseline characteristics (table 1), maximum flow rate was significantly better in continent patients (median $31 \mathrm{vs.} 11 \mathrm{ml} / \mathrm{s}, \mathrm{p}=0.021$ ). However, both groups exhibited no differences in the amount of residual urine.

In real-time MRI, before voiding, the neobladder neck was positioned $+5.4 \mathrm{~mm}$ (median; range +2.0 to $+9.0 \mathrm{~mm}$ ) above the PCL in continent patients. In comparison, in incontinent patients, the neobladder neck was positioned significantly lower $(+2 \mathrm{~mm}$, range 0.0 to $+4.0 \mathrm{~mm})(\mathrm{p}=$ 0.012) (fig. 1). 
Fig. 1. a Continent patient before voiding (left) and at end of voiding (right). b Incontinent patient before voiding (left) and at end of voiding (right). Red $=$ PCL; green $=$ orthogonal distance neobladder neck to PCL.
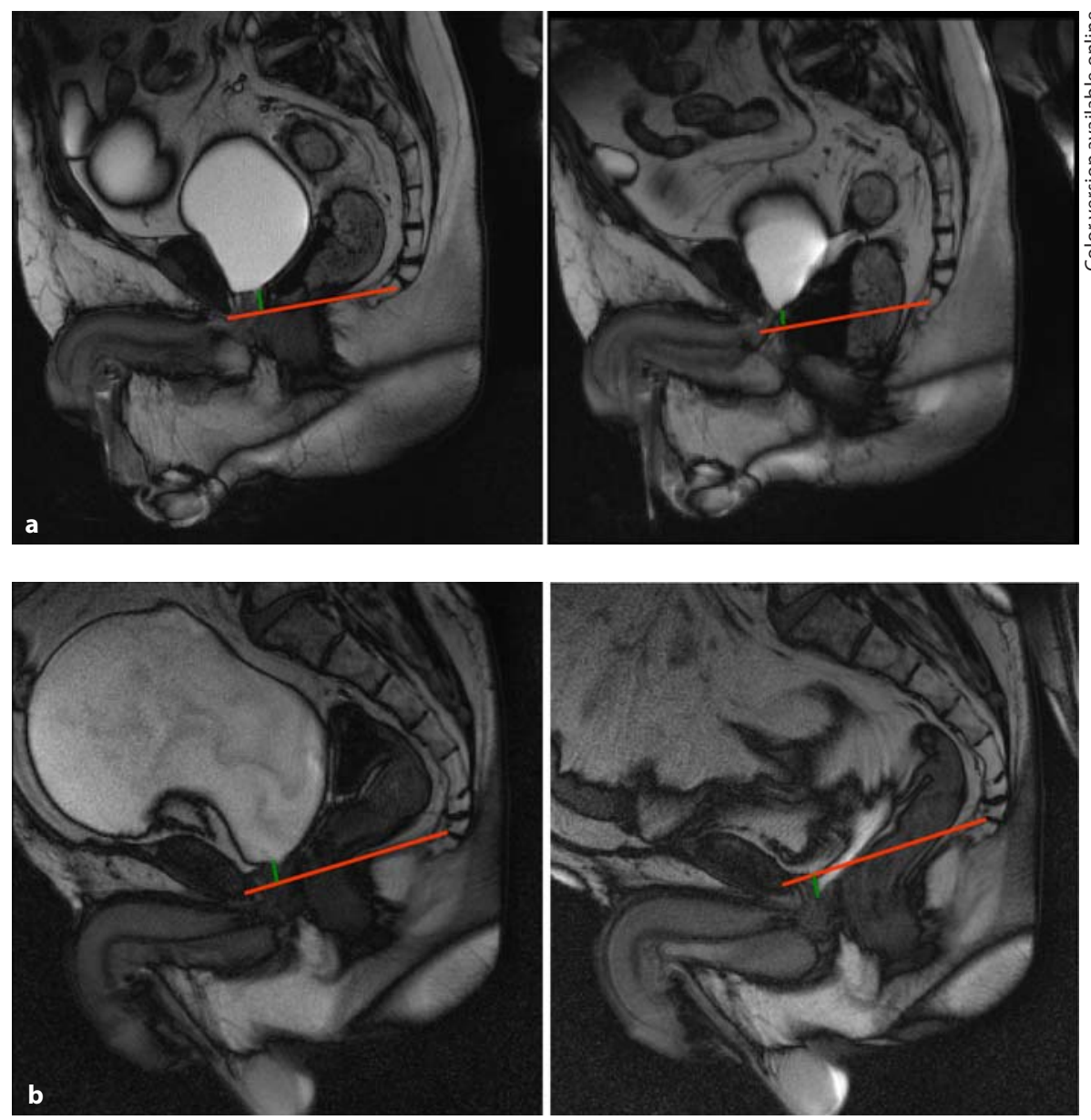

Table 1. Patients' baseline characteristics (security pad $=0.5$ pad)

\begin{tabular}{llll}
\hline Parameter & $\begin{array}{l}\text { Continent patients } \\
(\mathrm{n}=7)\end{array}$ & $\begin{array}{l}\text { Incontinent patients } \\
(\mathrm{n}=7)\end{array}$ & $\begin{array}{l}\text { Significance } \\
\mathrm{p}\end{array}$ \\
\hline Age at MRI, years & $53-74(66.9 / 69.0)$ & $54-83(68.6 / 69.0)$ & 0.798 \\
Age at neobladder, years & $51-72(65.4 / 68.0)$ & $52-80(66.6 / 67.0)$ & 0.949 \\
BMI at neobladder, kg/m 2 & $25-31(27.6 / 28.0)$ & $22-29(26.0 / 27.0)$ & 0.367 \\
Residual urine, ml & $0-30(4.3 / 0)$ & $0-40(17.9 / 10)$ & 0.086 \\
Capacity of neobladder, ml & $270-526(360 / 345)$ & $280-510(371 / 370)$ & 0.821 \\
Maximum flow rate, ml/s & $12-66(34.0 / 31.0)$ & $7-31(15.4 / 11.0)$ & 0.021 \\
Urine loss in 1-hour pad test, g & $0-2(0.9 / 1.0)$ & $25-170(59.9 / 52.0)$ & 0.002 \\
Daily pad use, pads/day & $0-0.5(0.1 / 0.0)$ & $1-5(3.0 / 3.0)$ & 0.001 \\
Nightly pad use, pads/night & $0-1.0(0.6 / 0.5)$ & 3 patients: $1-3$ diapers/night & 4 patients: condom catheter \\
& & &
\end{tabular}

Values denote ranges with mean/median values in parentheses. 


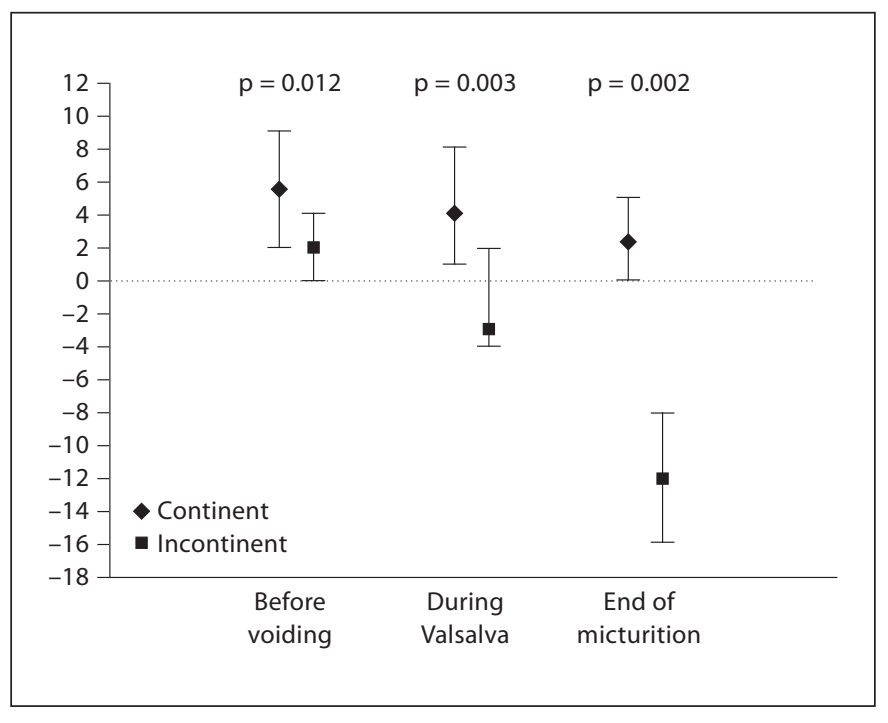

Fig. 2. Quantitative comparison of the neobladder neck position between continent and stress incontinent patients.

During the Valsalva maneuver, the neobladder neck moved caudally relative to the PCL with distances of $+4.0 \mathrm{~mm}(+1.0$ to $+8.0 \mathrm{~mm})$ in continent patients and $-3.0 \mathrm{~mm}(-4.0$ to $+2.0 \mathrm{~mm})$ in stress incontinent patients $(\mathrm{p}=0.003)$.

When micturition was initiated, relaxation of the pelvic floor (i.e. descending of the levator ani muscle) with a simultaneous descent of the external sphincter and neobladder neck in relation to the PCL was observed.

At the end of micturition, the neobladder neck had descended downwards to $+2.3 \mathrm{~mm}(0.0$ to $+5.0 \mathrm{~mm})$ above the PCL in continent patients (fig. 1). In incontinent patients, the neobladder neck had moved even further below the PCL with distances of $-12 \mathrm{~mm}(-16.0$ to $-8.0 \mathrm{~mm}$ ) relative to the PCL $(\mathrm{p}=0.002)$ (fig. 1$)$. The quantitative comparison of the neobladder neck position between continent and stress incontinent patients is presented in figure 2 .

In summary, the neobladder neck descended below the PCL in daytime stress incontinent patients during micturition and the Valsalva maneuver. The extent of the neobladder neck movement during micturition and Valsalva was also significantly higher in incontinent patients and differences in the anatomical position of the neobladder during Valsalva and micturition was significantly higher in stress incontinent patients $(\mathrm{p}=0.002$ vs. $\mathrm{p}=$ $0.001)$, respectively. In contrast, in continent patients the neobladder neck stayed above the PCL during micturition and Valsalva.

\section{Discussion}

There is relatively little knowledge on mechanism of continence in orthotopic ileal neobladder. It is known that daytime stress incontinence may arise from reduced urethral outlet resistance in combination with low neobladder capacity, reduced compliance, or elevated neobladder pressure [7-10]. However, similar urodynamic results can be demonstrated for the $\mathrm{W}$-shaped neobladder compared to naive bladder [11] and it remains unclear why some neobladder patients show daytime SUI despite a good sphincter function and a W-shaped pouch. A possible correlation between SUI and the position of the neobladder has been suggested.

Postoperative imaging studies are established for the evaluation of postsurgical complications and follow-up [12-15]. Several imaging studies used computed tomography and static MRI to evaluate postoperative anatomy and functional aspects of the neobladder [9, 15-18]. One of these studies showed that the location of the neobladder neck in the pelvis has an important impact on voiding: in patients showing good void competence the neobladder neck is located at the most caudal portion of the pouch [17]. Moreover, the position of the neobladder relative to the right femur head showed a linear correlation to continence status [16]. In addition, a wide funneling seems to be necessary for successful micturition [9]. However, all these imaging studies evaluated single static MRI images and no real-time sequences. Therefore, the dynamics of the voiding process remained elusive.

Dynamic magnetic resonance studies were first introduced for postoperative assessment [19]. Later, the micturition process of naive bladders was studied via MRI [20, 21]. Finally, Muto et al. [4] used for the first time real-time MRI to compare dynamics of the bladder with naive bladder with that of continent patients after neobladder. The findings showed that the neobladder $(n=5)$ rotated and moved more dynamically during the voiding process compared to patients with naive bladder $(n=5)$.

In the present study, we evaluated for the first time via real-time MRI differences in neobladder position with reference to the pelvic floor between continent and daytime stress incontinent patients with neobladders. Both groups were selected such that the patients did not differ with regard to their preoperative or surgery-related characteristics. Postoperatively, the two groups differed only in their daytime continence status and the significantly higher maximum flow rate in the continent group.

Our MRI-based analysis showed that the neobladder in incontinent patients exhibited significantly more mo- 
bility during micturition and the Valsalva maneuver, and was positioned significantly lower in the pelvis compared to continent patients. These results support the hypothesis that a 'stable' neobladder and neobladder neck are important factors for continence in patients with neobladder. Our finding that incontinent patients are characterized by a significantly lower position of the neobladder in the pelvis is in line with the results of Crivellaro et al. [16]. Altogether, these results are well in agreement with the integral theory of Petros, according to which pelvic floor integrity is a prerequisite for continence [22]. In incontinent patients with $\mathrm{W}$-shaped neobladder, the more pronounced bladder descensus - as observed by MRI - may be indicative of pelvic floor dysfunction due to damage of the supporting tissue or the nerve system. It can be hypothesized that this dysfunction is causative for incontinence in these patients despite good sphincter function. Conversely, in continent neobladder patients, the disintegrated supporting tissue may be compensated by pelvic floor muscle activity which is indicated in MRI by a less pronounced descending neobladder. In fact, the higher flow rate observed in the continent patient group may be - besides a potential better relaxation of the pelvic floor muscles in continent men - another indication for a generally higher muscular strength in the abdominal and pelvic floor region of continent patients.

Despite these conclusive findings, this study is limited by a rather small number of patients. Therefore, our initial findings have to be confirmed in a larger patient group and also compared to preoperative findings by further studies. In addition, the MRI was performed in a supine position. However, in an upright position we expect even more distinct results due to the effect of gravity.

\section{References}

1 Stenzl A CN, De Santis M, Kuczyk M, Merseburger AS, Ribal MJ, Sherif A, Witjes JA: Guidelines on bladder cancer - muscle-invasive and metastatic; in Arnheim AG (ed): EAU guidelines. Arnheim, European Association of Urology, 2010, pp 28-37.

$\checkmark 2$ El Sayed RF, El Mashed S, Farag A, Morsy MM, Abdel Azim MS: Pelvic floor dysfunction: assessment with combined analysis of static and dynamic MR imaging findings. Radiology 2008;248:518-530.

$\checkmark 3$ Lienemann A, Sprenger D, Janssen U, Grosch E, Pellengahr C, Anthuber C: Assessment of pelvic organ descent by use of functional cine-MRI: which reference line should be used? Neurourol Urodyn 2004;23:33-37.

-4 Muto S, Kamiyama Y, Ide H, Okada H, Saito K, Nishio K, Tokiwa S, Kaminaga T, Furui S, Horie S: Real-time MRI of orthotopic ileal neobladder voiding: preliminary findings. Eur Urol 2008;53:363-369.

5 Abrams P, Blaivas JG, Stanton SL, Andersen JT: The standardisation of terminology of lower urinary tract function. The International Continence Society Committee on Standardisation of Terminology. Scand J Urol Nephrol 1988;114:5-19.

6 Abrams P, Cardozo L, Fall M, Griffiths D, Rosier P, Ulmsten U, Van Kerrebroeck P, Victor A, Wein A: The standardisation of terminology in lower urinary tract function: report from the standardisation sub-committee of the International Continence Society. Urology 2003;61:37-49.

7 Steers WD: Voiding dysfunction in the orthotopic neobladder. World J Urol 2000;18: 330-337. $\checkmark 8$ Koraitim MM, Atta MA, Foda MK: Orthotopic bladder substitution in men revisited: identification of continence predictors. J Urol 2006;176:2081-2084.

-9 Schrier BP, Laguna MP, van der Pal F, Isorna S, Witjes JA: Comparison of orthotopic sigmoid and ileal neobladders: continence and urodynamic parameters. Eur Urol 2005;47: 679-685.

10 Chen Z, Lu G, Li X, Li X, Fang Q, Ji H, Yan J, Zhou Z, Pan J, Fu W, Li W, Xiong E, Song B: Better compliance contributes to better nocturnal continence with orthotopic ileal neobladder than ileocolonic neobladder after radical cystectomy for bladder cancer. Urology 2009;73:838-844.

11 Marim G, Bal K, Balci U, Girgin C, Dincel C: Long-term urodynamic and functional analysis of orthotopic 'W' Ileal neobladder following radical cystectomy. Int Urol Nephrol 2008;40:629-636.

12 Sassi C, Santilli L, Concetti S, Schiavina R, Amadori E, Severini E, Martorana G, Battista G: Three-dimensional computed tomography of the orthotopic ileal neobladder reconstruction: normal and abnormal findings. Urol Int 2009;82:301-305.

3 Sudakoff GS, Guralnick M, Langenstroer P, Foley WD, Cihlar KL, Shakespear JS, See WA: CT urography of urinary diversions with enhanced CT digital radiography: preliminary experience. AJR 2005;184:131-138.

14 Catala V, Sola M, Samaniego J, Marti T, Hu guet J, Palou J, De La Torre P: CT findings in urinary diversion after radical cystectomy: postsurgical anatomy and complications. Radiographics 2009;29:461-476.
15 Gogus C, Turkolmez K, Fitoz S, Ozden E, Yagci C, Gogus O: Three-dimensional computerized tomography in follow-up of patients with urinary diversion. Int Urol Nephrol 2005;37:739-742.

16 Crivellaro S, Mami E, Wald C, Smith JJ, Kocjancic E, Stoffel J, Bresette J, Libertino JA: Correlation between urodynamic function and $3 \mathrm{D}$ cat scan anatomy in neobladders: does it exist? Neurourol Urodyn 2009;28: 236-240

-17 Porru D, Madeddu G, Campus G, Montisci I, Caddemi G, Scarpa RM, Usai E: Urodynamic analysis of voiding dysfunction in orthotopic ileal neobladder. World J Urol 1999;17: 285-289.

18 Caproni N, Ligabue G, Mami E, Torricelli P: Reconstructed urinary bladder following radical cystectomy for bladder cancer. Multidetector CT evaluation of normal findings and complications. Radiol Med 2006;111: 1134-1145

19 Tempany CM, Masoudi FA, Marshall FF: The use of dynamic magnetic resonance imaging to evaluate orthotopic continent urinary diversion. Urology 1995;45:886-892

-20 Simmons A, Williams SC, Craggs M, Andrew C, Gregory L, Allin M, Mundy A, Leaker B: Dynamic multi-planar EPI of the urinary bladder during voiding with simultaneous detrusor pressure measurement. Magn Reson Imaging 1997;15:295-300.

$>21$ Hocaoglu Y, Gozzi C, Mayer ME, Roosen A, Stief CG, Bauer RM: Real-time MRI: anatomical changes during physiological voiding in men. Eur Urol Suppl 2010;9:104-105.

-22 Petros PE, Woodman PJ: The integral theory of continence. Int Urogynecol J 2008;19:3540 . 